\title{
Ultrafiltración de Caldos de Fermentación
}

Carlos Orozco, Gerardo Albarrán, Rosa Esteban y Sergio García

Instituto Politécnico Nacional, Departamento de Bioingeniería, Unidad Profesional Interdisciplinaria de Biotecnología, Av. Acueducto s/n, Col. Barrio La laguna Ticomán, Delegación G.A. Madero, México, D.F.-México (e-mail: tepoztlan61@yahoo.com.mx)

\section{Resumen}

Se estudió la microfiltración tangencial y ultrafiltración para concentrar caldos de levadura de Phaffia rhodozyma, la que es empleada para la producción del colorante astaxantina. Se emplearon cartuchos de microfiltración y ultrafiltración para evaluar el efecto de las variables de operación sobre el flujo de permeado. Se trabajó con presiones transmembrana de 35 a $210 \mathrm{kPa}$, flujos de alimentación de 7 a $30 \times 10^{-6} \mathrm{~m}^{3} / \mathrm{s}$, pH de 4 a 8 , y temperatura de 303 a $323 \mathrm{~K}$. El flujo más alto alcanzado en la microfiltración fue de $13 \times 10^{-6} \mathrm{~m} / \mathrm{s}$, observándose la disminución de éste si el $\mathrm{pH}$ aumenta; mientras que para la ultrafiltración el flujo más alto fue de $17 \times 10^{-6} \mathrm{~m} / \mathrm{s}$. En ambos casos la concentración de levadura máxima alcanzada fue de $250 \mathrm{~kg} / \mathrm{m}^{3}$. Los resultados obtenidos muestran que la concentración de caldos de fermentación de Phaffia rhodozyma puede llevarse a cabo tanto por microfiltración tangencial como por ultrafiltración.

Palabras claves: microfiltración, ultrafiltración, levadura, Phaffia rhodozyma, fermentación

\section{Ultrafiltration in Fermentation Broths}

\begin{abstract}
Tangential microfiltration and ultrafiltration were studied to concentrate Phaffia rhodozym yeast broths, yeast that is used in production of astaxantine colorant. Microfiltration and ultrafiltration cartridges were used to study the effect of the operating variables on the permeated flux. The values used were: transmembranal pressures from 35 to $210 \mathrm{kPa}$, feeding flows from 7 a $30 \times 10^{-6} \mathrm{~m}^{3} / \mathrm{s}, \mathrm{pH}$ from 4 to 8; and temperature from 303 to $323 \mathrm{~K}$. The highest flux for microfiltration was $13 \times 10^{-6} \mathrm{~m} / \mathrm{s}$, observing that concentration decreases when the $\mathrm{pH}$ increases. For ultrafiltration the maximum flux was $17 \times 10^{-6} \mathrm{~m} / \mathrm{s}$. In both cases the maximum yeast concentration was $250 \mathrm{~kg} / \mathrm{m}^{3}$. The results show that concentration of Phaffia rhodozym yeast broths can be done using tangential microfiltration or ultrafiltration.
\end{abstract}

Keywords: ultrafiltration, Phaffia rhodozyma, yeast broths, microfiltration, fermentation 


\section{INTRODUCCIÓN}

La producción de salmones y trucha arco iris criados en granjas acuícolas se ha venido incrementando; en 1991 el 30 \% de salmón consumido provenía de granjas. La astaxantina es el pigmento responsable de la tonalidad rojiza característica de la carne de estos peces que al ser incapaces de sintetizarla la tienen que consumir a partir de formulaciones alimenticias comerciales. Se emplea de 35 a $75 \mathrm{mg}$ de astaxantina por kilogramo de alimento para peces y representa del 10 al $15 \%$ del costo del alimento.

Prácticamente toda la astaxantina consumida a nivel mundial es producida por síntesis química; el precio del colorante comercial, en 1991, fue de dos mil dólares americanos por kilogramo. La FDA ha prohibido una serie de colorantes sintéticos, los colorantes de origen natural gozan de amplio reconocimiento, razón por la cual se han desarrollado estudios para encontrar una vía natural de producción de astaxantina .

Existen distintas fuentes naturales de astaxantina: fitoplancton, ciertos crustáceos, algunas algas y ciertos microorganismos. La fuente microbiana representa una alternativa, sobre todo, cuando la tecnología de fermentaciones se encuentra desarrollada, en cuyo caso solo queda por encontrar el mejor microorganismo productor, las condiciones de fermentación y el proceso de recuperación y purificación de la astaxantina (Fleno et al., 1994).

Un microorganismo que ha mostrado potencial de ser utilizado para la producción de astaxantina es la levadura Phaffia rhodozyma; aquí cabe mencionar que en nuestra escuela se ha estado trabajando con el proceso de fermentación de este microorganismo para la obtención de astaxantina.

Se sabe que el contenido específico de astaxantina en cepas silvestres $P$. rhodozyma suele ser de $400 \mathrm{ppm}$ mientras que en cepas mutantes puede llegar hasta $3000 \mathrm{ppm}$. Se ha reportado que cuando se ocupa la levadura rupturada en la formulación del alimento, el pigmento se deposita más eficientemente que cuando la célula está entera. Dentro de los métodos reportados para la ruptura celular están el rompimiento mecánico y tratamiento químico, teniendo un mejor rendimiento el primero. Finalmente el concentrado celular rupturado o no, se somete a un proceso de secado para obtener el producto en estado sólido.

Así, para obtener el concentrado celular, una vez finalizada la fermentación, las operaciones como la microfiltración tangencial y la ultrafiltración son las recomendadas. Éstas han venido desplazando a las operaciones tradicionales como la centrifugación y filtración convencional porque reducen los tiempos de operación y el costo de inversión del equipo es menor.

Con base en esto, se propone el presente trabajo para estudiar la microfiltración tangencial y ultrafiltración para concentrar caldos de fermentación de Phaffia rhodozyma.

\section{METODOLOGÍA}

Los caldos de fermentación de Phaffia rhodozyma se obtuvieron de su cultivo en un fermentador encamisado tipo tanque agitado con capacidad de $0,015 \mathrm{~m}^{3}$ de volumen de operación (Bowen et al., 1996). Se elaboró una curva tipo de concentración celular vs. absorbancia la que sirvió para calcular el nivel de concentración en las suspensiones después de cada operación de filtración tangencial.

Los primeros experimentos se realizaron en un cartucho de microfiltración tangencial de laboratorio (diámetro de poro de $0.2 \mu \mathrm{m}$ y un área de membrana de $0.042 \mathrm{~m}^{2}$ ), donde se investigaron variables como flujo de alimentación: 7 a $30 \times 10^{-6} \mathrm{~m}^{3} / \mathrm{s}$; presión transmembrana: 35 a $210 \mathrm{kPa}$; pH : 4.0 a 8.0 ; y temperatura de 303 a $323^{\circ} \mathrm{K}$. Se evaluó el efecto de estas variables en el flux asi como también el efecto del grado de concentración de los caldos de fermentación en el desempeño de las membranas (Yeh et al., 2003). Este último se determinó con base en la concentración celular y el volumen de filtrado eliminado. Esta misma evaluación se efectuó empleando ahora un cartucho de ultrafiltración de laboratorio con un corte molecular de $100 \mathrm{kDa}$ y un área de membrana de $0.042 \mathrm{~m}^{2}$ (Orozco et al., 2003). 
A continuación y a manera de glosario se definen los siguientes términos para una mayor claridad del procedimiento experimental (ver Fig. 1). El cartucho es el módulo donde se encuentra la membrana arreglada en forma de fibras huecas. El flujo de alimentación es el caudal de caldo de fermentación que se suministra a los cartuchos de microfiltración y ultrafiltración. El flux es el caudal de filtrado obtenido del cartucho dividido entre el área de la membrana. La presión transmembrana se estima como el promedio de las presiones de entrada y salida del cartucho y a este promedio se le resta la presión del filtrado. El término "corte molecular" de 100 kDa indica que la membrana de ultrafiltración puede retener moléculas o partículas que tengan un tamaño igual ó mayor a este valor.

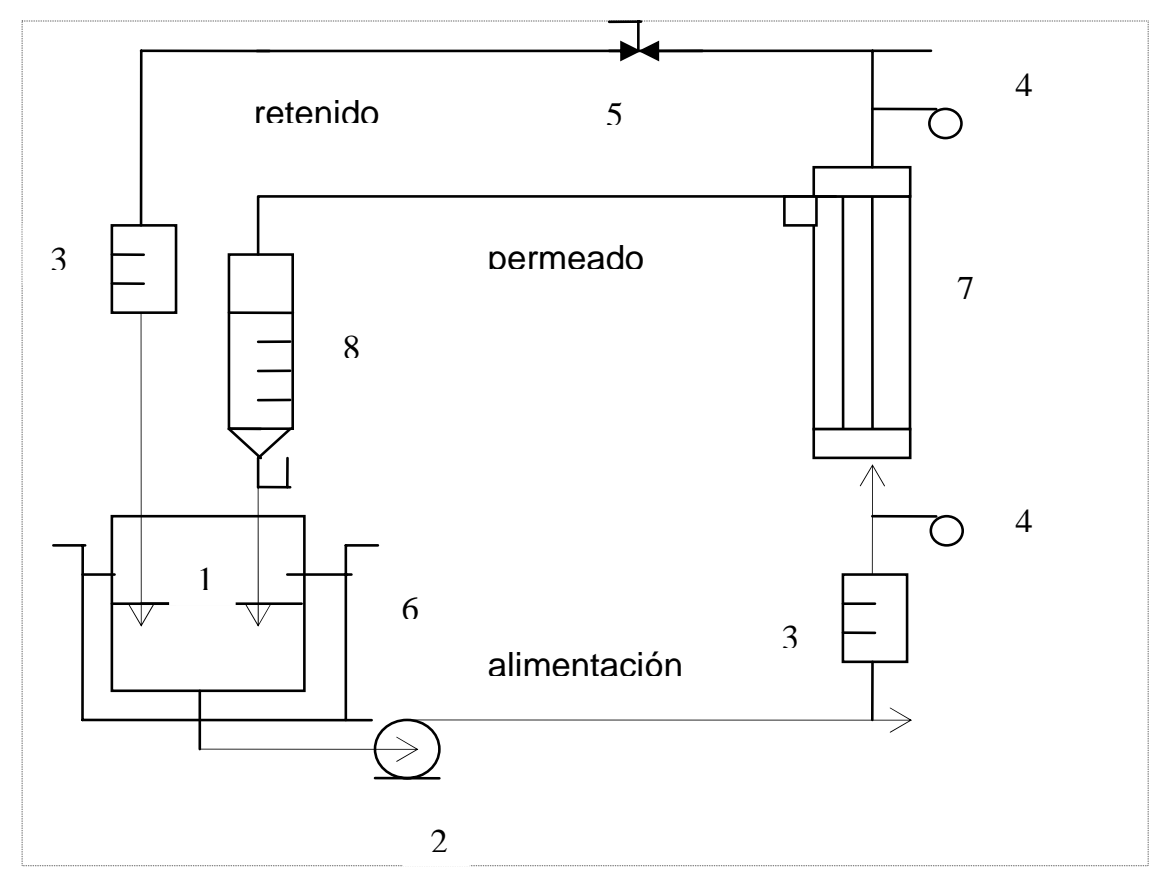

Fig. 1: Equipo de micro y ultrafiltración. (1) tanque de alimentación, (2) bomba peristáltica, (3) rotámetro, (4) manómetro, (5) válvula de contra presión, (6) termostato, (7) módulo de fibra hueca y (8) probeta.

\section{RESULTADOS Y DISCUSIÓN}

Primeramente se trabajaron los cartuchos usando agua como fluido para conocer cuáles eran las condiciones máximas de presión transmembrana y flujo de alimentación que permitía el equipo.

Los resultados se muestran en la Fig. 2, donde se observa que el equipo permite trabajar hasta 210 $\mathrm{kPa}$ de presión transmembrana, aunque las especificaciones del cartucho recomiendan que se trabaje hasta $140 \mathrm{kPa}$ para mayor tiempo de vida del mismo.

También se ve que el flux en el cartucho de microfiltración son aproximadamente 9 veces mayores que los obtenidos con el cartucho de ultrafiltración a cualquier presión de trabajo. Esto era de esperarse porque el diámetro de poro de la microfiltración es superior al de ultrafiltración en varios órdenes. Con estos resultados es posible obtener la resistencia hidráulica de la membrana la cual es simplemente el inverso de la pendiente del gráfico anterior. Para microfiltración la resistencia es de $0.45 \times 10^{6} \mathrm{kPa} . \mathrm{s} / \mathrm{m}$, mientras que para la ultrafiltración la resistencia es hasta ocho veces mayor que la obtenida para microfiltración. Estos resultados son congruentes con los valores de flux obtenidos en ambos cartuchos (Yeh et al, 2003).

Para la concentración de los caldos de fermentación por microfiltración tangencial se realizó toda una serie de pruebas para encontrar las mejores condiciones de presión transmembrana y flujo de alimentación en los que se obtuvieran los valores más altos del flux. En la Fig. 3, se indican los resultados encontrados.

Los valores de flux son muchísimo más bajos que los obtenidos cuando se trabaja sólo con agua: bajan de 420 a $8 \times 10^{-6} \mathrm{~m} / \mathrm{s}$, en promedio, a $140 \mathrm{kPa}$. La segunda observación es que a partir de una 
concentración de levadura de $50 \mathrm{~kg} / \mathrm{m}^{3}$ el flux prácticamente permanece constante aunque se vaya incrementando la concentración (Naja et al., 2006). También se observa que cuando se trabaja a flujos de alimentación de solo $7 \times 10^{-6} \mathrm{~m}^{3} / \mathrm{s}$, se alcanzan los valores más bajos de flux aunque la presión se incremente de 30 a $210 \mathrm{kPa}$ (Frenander y Jonsson, 1996).

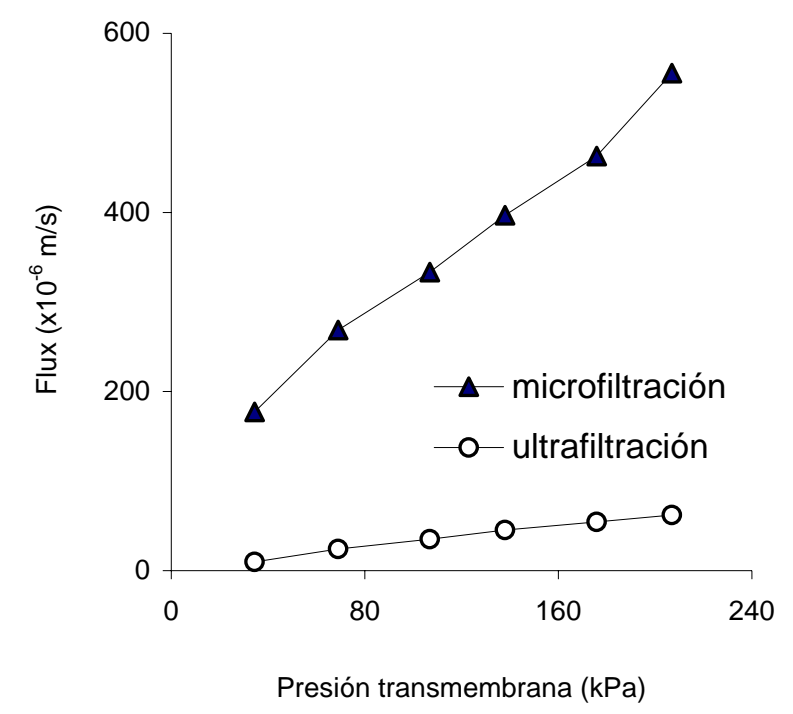

Fig. 2: Desempeño de los cartuchos con agua

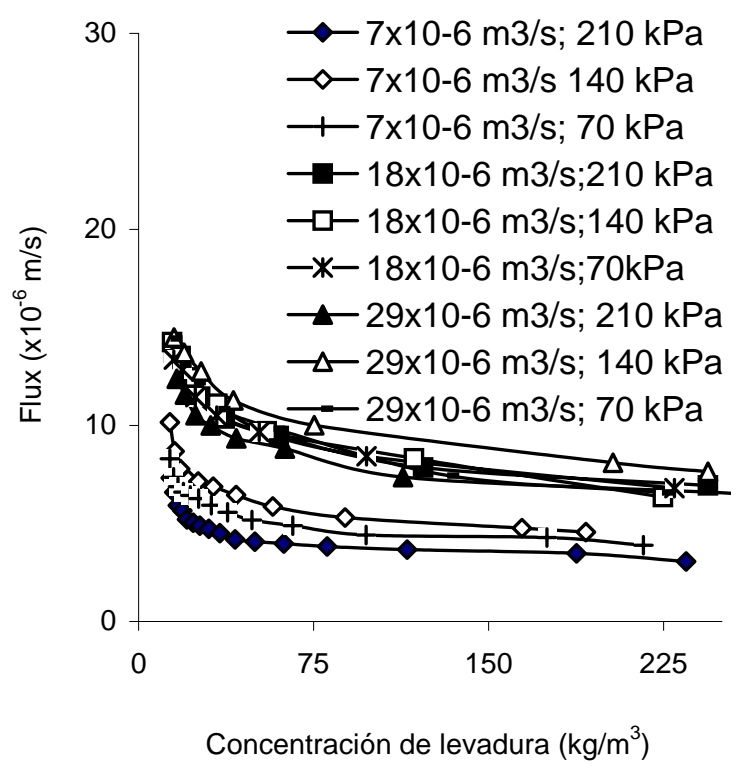

Fig 3: Efecto del flujo de alimentación y presión transmembrana en la microfiltración: $\mathrm{pH} 6.0$ y $303^{\circ} \mathrm{K}$

Cuando se incrementa el flujo de alimentación a $18 \times 10^{-6} \mathrm{~m}^{3} / \mathrm{s}$ se obtienen el flux más alto y a su vez el valor más alto se obtiene a $70 \mathrm{kPa}$, y no a $140 \mathrm{kPa}$ (Meireles et al., 2002). Es decir, el incremento de presión provoca una mayor "aglomeración" de la levadura en la superficie de la membrana lo cual no puede ser contrarrestado por el flujo de alimentación causando una disminución en el flux (Graves et al., 2006).

Inclusive aumentando el flujo de alimentación de 18 a $29 \times 10^{-6} \mathrm{~m}^{3} / \mathrm{s}$ no es posible incrementar el flux (a $210 \mathrm{kPa}$ ) y el equipo no permite trabajar mayores flujos de alimentación. Esto significa que el flujo de alimentación asi como la presión de trabajo tienen un impacto significativo sobre el desempeño de la membrana (Liew et al., 1995). 
En la Fig. 4, se presentan los resultados obtenidos de la concentración de los caldos por ultrafiltración. Se observa que el flux en promedio es mucho más bajo que el encontrado con agua: baja de 70 a $10 \times 10^{-6} \mathrm{~m} / \mathrm{s}$ trabajando a $140 \mathrm{kPa}$ de presión transmembrana.

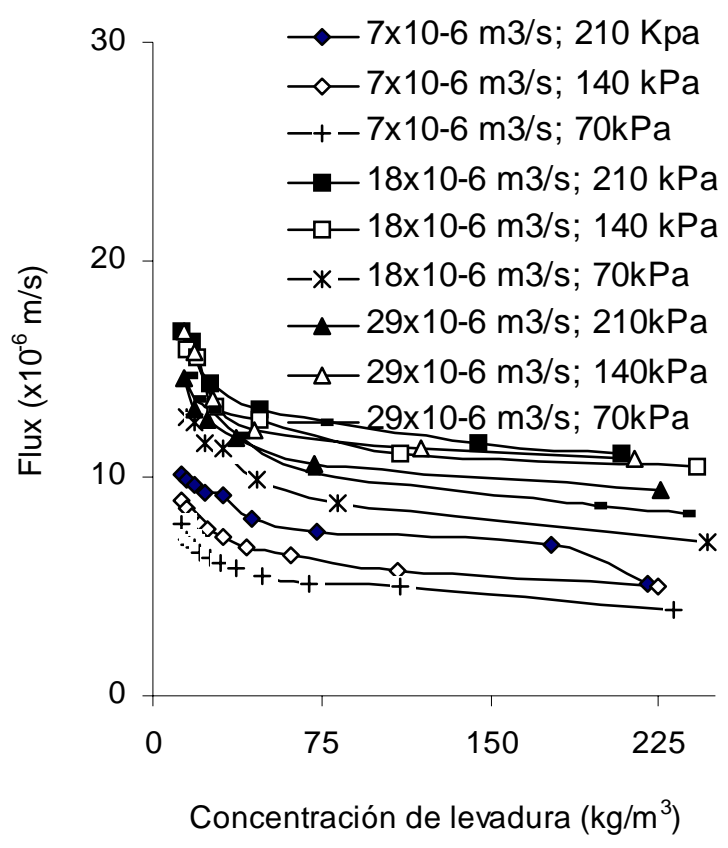

Fig. 4: Efecto del flujo de alimentación y presión transmembrana en la ultrafiltración: pH 6.0 y 303 K

El flux es prácticamente una línea horizontal en el intervalo de concentraciones de 25 a $200 \mathrm{~kg} / \mathrm{m}^{3}$. La tendencia es que a la misma presión el flux se incrementa si se aumenta el flujo de alimentación (Yeh et al., 2003). Las condiciones que maximizan el flux fueron: trabajar a una presión transmembrana de $210 \mathrm{kPa}$ y un flujo de alimentación de $18 \times 10^{-6} \mathrm{~m}^{3} / \mathrm{s}$.

\section{Efecto del $\mathrm{pH}$}

Una vez que se encontraron las condiciones de presión transmembrana y flujo de alimentación para la concentración de los caldos por microfiltración y ultrafiltración, se procedió a investigar el efecto del $\mathrm{pH}$. Los resultados se encuentran resumidos en la Fig. 5, en la cual se exponen los datos de la microfiltración tangencial y la ultrafiltración.

Se observa que el flux obtenido con el cartucho de microfiltración disminuye a medida que el valor del pH se incrementa (Graves et al., 2006) y son inferiores a los obtenidos con la ultrafiltración. Así, el mayor flux se consigue con el cartucho de ultrafiltración trabajando a un pH de 5 ó 6.

Algunos autores explican que el flux en la ultrafiltración es mayor que en la microfiltración debido a que la levadura no puede penetrar en los orificios de la membrana mientras que sí lo pueden hacer en la membrana de microfiltración, causando en esta última una obstrucción que provoca la disminución del flux (Patel et al., 1987).

La explicación que puede darse acerca de que el flux sea mayor a valores de pH de 5 ó 6 es que la superficie de la levadura posea una carga neta que provoque cierta repulsión entre ellas y que esto se vea reflejado en una menor tendencia a aglomerarse en la superficie de la membrana dejando mayores espacios entre las células adyacentes lo que finalmente resulta en mayores valores de flux (Persson et al., 2003).

\section{Efecto de la temperatura}

Finalmente se estudió el efecto de la temperatura en la concentración de caldos de levadura obteniéndose los resultados resumidos en la Fig. 6. La temperatura tiene un efecto directo en el flux 
y a una temperatura de $323^{\circ} \mathrm{K}$ se obtienen los mayores valores de flux. También se puede observar que el flux obtenido en la ultrafiltración es mayor que el de la microfiltración tangencial.

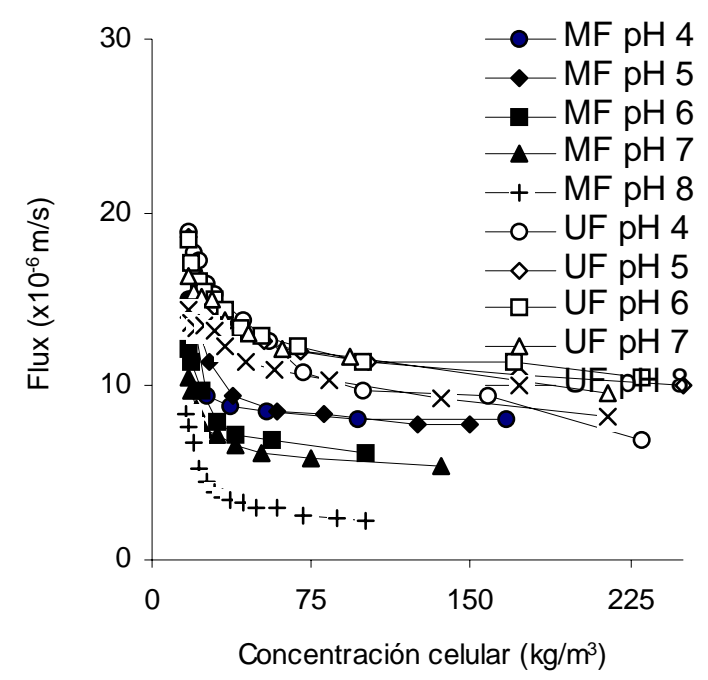

Fig. 5: Efecto del pH en la microfiltración y ultrafiltración: $140 \mathrm{kPa}, 303^{\circ} \mathrm{K}$ y $18 \times 10^{-6} \mathrm{~m}^{3} / \mathrm{s}$

La literatura explica que al aumentar la temperatura se incrementa la transferencia de materiales de regreso al seno de la corriente de alimentación, es decir, disminuye la acumulación de levadura en la superficie de la membrana originando un mayor flux (Bell y Davies, 1987).

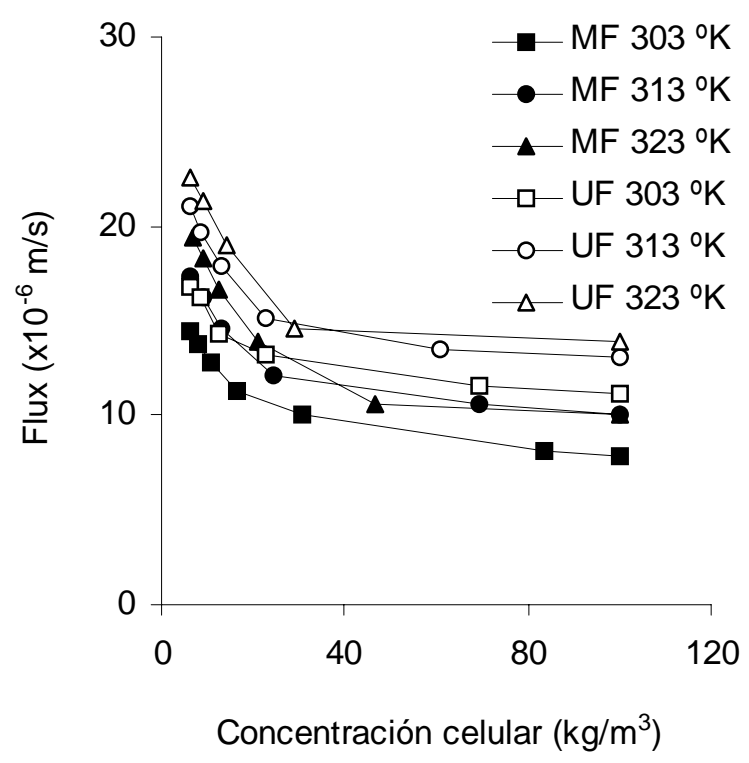

Fig. 6: Efecto de la temperatura en la microfiltración y ultrafiltración: $140 \mathrm{kPa}, \mathrm{pH} 6$ y $18 \times 10^{-6} \mathrm{~m}^{3} / \mathrm{s}$

\section{CONCLUSIONES}

Los resultados obtenidos demuestran que la concentración de caldos de fermentación de Phaffia rhodozyma puede llevarse a cabo tanto por microfiltración tangencial como por ultrafiltración. Si se utiliza la microfiltración el mayor flux se obtiene trabajando a los máximos valores de flujo de alimentación y temperatura, a un pH de 5 ó 6 y a una presión entre 70 y $140 \mathrm{kPa}$. Cuando se emplea la ultrafiltración la maximización del flux se alcanza operando a los máximos valores permitidos de flujo de alimentación, temperatura y presión transmembrana, y también a un pH de 5 ó 6 .

Así, se recomienda la ultrafiltración para la concentración de los caldos de fermentación porque el flux obtenido es más elevado que el alcanzado en la microfiltración, lográndose en ambas operaciones concentraciones celulares de hasta $250 \mathrm{~kg} / \mathrm{m}^{3}$. 


\section{AGRADECIMIENTOS}

Este proyecto fue financiado por Secretaría de Investigación y Posgrado del Instituto Politécnico Nacional.

\section{REFERENCIAS}

Bell, D.J. y R.J. Davies; Cell harvesting of oleaginous yeast by cross-flow filtration, Biotechnology and Bioengineering, Vol XXIX, 1176-1178 (1987).

Bowen Fontana, J.D. y otros cuatro autores; Culture of the astaxanthinogenic yeast Phaffia rhodozyma in low-cost media, Appl. Biochem. Biotechnol., 57-58: 413-422 (1996)

Fleno, B. y otros cuatro autores; Astaxanthin producing yeast cells, methods for their preparation and their use, US 5.356.810 (1994).

Frenander, U. y A.S. Jonsson; Cell harvesting by cross-flow microfiltration using a shear-enhanced module, Biotechnology and Bioengineering, 52:397-403 (1996).

Graves, K., G. Rozeboom, M. Heng y Ch. Glatz; Broth Conditions Determining Specific Cake Resistance During Microfiltration of Bacillus subtilis, Biotechnology and Bioengineering, 94:346-352 (2006).

Liew, M.K., A.G. Fane y P.L. Rogers; Hydraulic resistance and fouling of microfilter by Candida utilis in fermentation broth, Biotechnology and Bioengineering, Vol. 48, 108-117 (1995).

Meireles, M., M. Clifton y P. Aimar; Filtration yeast suspensions: experimental observations and modelling of dead-end filtration with a compresible cake, Desalination, 147: 19-23 (2002).

Naja, G., B. Volesky y A. Schnell; Performance análisis of an integrated tangencial microfilterfermenter, Chem Technol Biotechnol, 81:648-658 (2006).

Orozco, A.C., R.D Vidal, S.S. García y C.L Ordaz; Concentración de suspensiones de levadura por filtración tangencial, Tecnología de alimentos, 38 (2), 7-17 (2003).

Patel, P.N., M. Mehaia y M. Cheryan; Cross-flow membrane filtration of yeast suspensions. Journal of Biotechnology, 5: 1-16 (1987).

Persson, A., A. Jonsson y G. Zacchi; Transmission of BSA during cross-flow microfiltration: Influence of $\mathrm{pH}$ and salt concentration, Journal of Membrane Science, 95:125-134 (2003).

Yeh, H.M., H.P. Wu y J.F. Dong; Effects of design and operating parameters on the declination of permeate flux for membrane ultrafiltration along hollow - fiber modules, Journal of Membrane Science, $213: 33$ - 44 (2003). 
